\title{
The PD-1/PD-L1 axis contributes to T-cell dysfunction in chronic lymphocytic leukemia
}

\author{
Davide Brusa, ${ }^{1}$ Sara Serra, ${ }^{1}$ Marta Coscia, ${ }^{2}$ Davide Rossi, ${ }^{3}$ Giovanni D’Arena, ${ }^{4}$ Luca Laurenti, ${ }^{5}$ Ozren Jaksic, ${ }^{6}$ \\ Giorgio Fedele, ${ }^{7}$ Giorgio Inghirami, ${ }^{8}$ Gianluca Gaidano, ${ }^{3}$ Fabio Malavasi, ${ }^{9}$ and Silvia Deaglio ${ }^{1}$
}

\begin{abstract}
${ }^{1}$ Human Genetics Foundation (HuGeF) and Department of Medical Sciences, University of Turin, Turin, Italy; ${ }^{2}$ Division of Hematology, Laboratory of Hematology Oncology, Center of Experimental Research and Medical Studies, San Giovanni Battista University Hospital, Turin, Italy; ${ }^{3}$ Division of Hematology, Department of Translational Medicine, Amedeo Avogadro University of Eastern Piedmont, Novara, Italy; ${ }^{4}$ Department of Onco-Hematology, IRCCS "Centro di Riferimento Oncologico della Basilicata," Rionero in Vulture, Italy; ${ }^{5}$ Institute of Hematology, Catholic University of the Sacred Heart, Rome, Italy; ' Department of Hematology, Dubrava University Hospital, Zagreb, Croatia; ${ }^{7}$ Department of Infectious, Parasitic and Immunomediated Diseases, Istituto Superiore di Sanità, Rome, Italy; ${ }^{8}$ Department of Molecular Biotechnology and Health Sciences, Center of Experimental Research and Medical Studies; and ${ }^{~}$ Laboratory of Immunogenetics, Department of Medical Sciences, University of Turin, Turin, Italy
\end{abstract}

Chronic lymphocytic leukemia is marked by profound defects in T-cell function. Programmed death-1 is a receptor involved in tumor-mediated immunosuppression through binding of the PD-L1 ligand. Multiparametric flow cytometry and immunohistochemistry were used to study PD-1/PD-L1 expression. Functional assays were used to determine the involvement of the PD-1/PD-L1 axis in T-cell responses. PD-1 expression by $\mathrm{CD}^{+}$and $\mathrm{CD}^{+} \mathrm{T}$ lymphocytes was significantly higher in 117 chronic lymphocytic leukemia patients than in 33 donors of a comparable age. $\mathrm{CD}^{+}$and $\mathrm{CD}^{+} \mathrm{T}$ lymphocytes from chronic lymphocytic leukemia patients displayed increased numbers of effector memory and terminally differentiated cells, respectively, when compared to controls. The number of effector memory $\mathrm{CD} 4^{+}$and terminally differentiated $\mathrm{CD} 8^{+}$lymphocytes positively associated with a more advanced stage of disease, treatment requirements and unfavorable genomic aberrations. Furthermore, leukemic lymphocytes expressed higher levels of PD-L1 than circulating B lymphocytes from normal donors. PD1 and PD-L1 surface expression spiked in proliferating $\mathrm{T}$ and $\mathrm{B}$ lymphocytes, suggesting that this interaction works efficiently in activated environments. Within chronic lymphocytic leukemia proliferation centers in the lymph node, CD4+/PD-1+ $\mathrm{T}$ lymphocytes were found to be in close contact with PD-L1+ chronic lymphocytic leukemia cells. Lastly, functional experiments using recombinant soluble PD-L1 and blocking antibodies indicated that this axis contributes to the inhibition of IFN- $\gamma$ production by $\mathrm{CD}^{+} \mathrm{T}$ cells. These observations suggest that pharmacological manipulation of the PD-1/PD-L1 axis may contribute to restoring T-cell functions in the chronic lymphocytic leukemia microenvironment.

\section{Introduction}

It is now largely accepted that chronic lymphocytic leukemia (CLL) fits best the model of a compartmentalized disease, with the proliferative component localized almost exclusively in lymphoid organs. ${ }^{1,2}$ Here, environmental interactions appear to fine tune the competence of leukemic cells to survive, grow and eventually become resistant to therapy. Distinct receptor-ligand pairs, as well as soluble molecules mediating crosstalk between CLL cells and stromal-derived elements, are attracting increasing attention as potential therapeutic targets. ${ }^{3,4}$ In addition, several lines of evidence indicate that CLL development and progression is accompanied by a progressive impairment of the host immune defenses. CLL is frequently associated with clinically manifest immune defects of the T-cell compartment, with abnormalities in the phenotype of $\mathrm{CD}^{+}$and $\mathrm{CD}^{+} \mathrm{T}$-cell subsets. A common finding is the accumulation of terminally differentiated effector memo- ry $\mathrm{T}$ cells, with a relative decrease of naive precursors. ${ }^{5,6}$ Furthermore, decreased $\mathrm{T}$-cell responses to mitogenic and $\mathrm{T}$ cell receptor-mediated stimulations have been described in patients with CLL. ${ }^{7,8}$ Histological studies of CLL lymph node (LN) samples have shown that within the proliferation centers (PC) (the counterpart of germinal centers ${ }^{9}$ ), leukemic cells are in close contact with a population of $\mathrm{CD}^{+} / \mathrm{CD} 25^{+} / \mathrm{Foxp} 3$ T lymphocytes..$^{10}$ In addition, the success of CLL engraftment and growth in an immunodeficient mouse was found to be selectively dependent on activated autologous $T$ lymphocytes, implying that this population is essential for in vivo neoplastic cell survival and proliferation. ${ }^{11}$

The mechanisms responsible for T-cell dysfunction in CLL remain unclear, even if several independent observations point to "frustrated" chronic antigen stimulation as a feature of the disease. In line with this hypothesis, T lymphocytes from CLL patients express markers of chronic activation, with an inversion of the normal CD4:CD8 ratio, highly reminis- 
cent of the clinical picture described for patients with chronic infections ${ }^{6,12} \mathrm{CD}^{+}$and $\mathrm{CD} 8^{+} \mathrm{T}$ lymphocytes from CLL patients show distinct gene profiles, ${ }^{13}$ with alterations in multiple genetic pathways, including the actin cytoskeleton. ${ }^{14}$ Functional studies confirmed that these $\mathrm{T}$ cells have defects in F-actin polymerization and immune synapse formation with antigen presenting cells, both essential steps in the generation of competent cytotoxic $T$ cells. The transmission of an immunosuppressive signal has been attributed to the interaction of inhibitory receptors expressed by CLL T lymphocytes (including CD200R, CD272 and CD279) with ligands expressed by leukemic cells (including CD200, CD270, CD274 and CD276). ${ }^{15}$

We investigated expression and functional significance of programmed death-1 (PD-1, CD279), a cell surface molecule involved in tumor-mediated suppression of activated immune cells through binding of the PD-L1 ligand, in a cohort of 117 CLL patients and compared them to agematched controls. Results provide evidence of an active crosstalk between PD-1 expressed by $\mathrm{CD}^{+}$and $\mathrm{CD} 8^{+}$subsets and PD-L1 expressed by the leukemic counterpart, operative within the PC in the CLL LN. Signaling through PD-1 contributes to blocking IFN- $\gamma$ secretion, with the final effect of a pronounced Th2 skewing of T-cell responses. These findings identify the PD-1/PD-L1 axis as an important component that contributes to dysfunctional interactions between leukemic CLL cells and host T lymphocytes.

\section{Design and Methods}

\section{Patients' samples}

Peripheral blood samples were obtained from 117 patients (51\% males) with a confirmed diagnosis of CLL (mean age 63 years). Thirty-three donors (54\% males) with no evidence of leukemia and comparable age (mean age 65 years) were kindly provided by the local blood bank (Online Supplementary Table S1). Blood was drawn after informed consent, in accordance with Institutional Guidelines and the Declaration of Helsinki. The study was approved by the Human Genetics Foundation (HuGeF) ethical committee. Peripheral blood mononuclear cell (PBMC) preparation and B-cell purification were as described in the Online Supplementary Appendix. Sample analyses included CD38 and ZAP-70 staining (cut-off positivity values of $20 \%$ ), IgVH mutational status (unmutated if $\geq 98 \%$ homology to the germ-line gene) and fluorescence in situ hybridization (FISH) for chromosomes 11, 12, 13 and 17 (Online Supplementary Table S1).

\section{FACS analyses}

Antibodies used for flow cytometry were anti-CD4-PE-Cy5, CD45RO-PE, -PD-L1-PE-Cy7, -IL4-FITC, -IFN- $\gamma$-PE (all from eBioscience, Milan, Italy), -CD8-FITC, -PD-1-PE-Cy7, -CD45RAAPC-Cy7 (Biolegend, Milan, Italy) and anti-CCR7-FITC (R\&D System, Milan, Italy). Data were acquired using a FACSCanto II cytofluorimeter (BD Biosciences, Milan, Italy) and processed with DIVA version 6.1.3 (BD Biosciences) and FlowJo version 9.01 softwares (TreeStar, Ashland, OR, USA). At least 10,000 events were analyzed for each sample.

\section{Immunohistochemistry and immunofluorescence microscopy}

Formalin-fixed, paraffin-embedded sections of LNs infiltrated by CLL cells $(n=20)$ were obtained from the Department of Pathology of the University of Turin, Italy, and from the
Department of Hematology of the Dubrava University Hospital, Zagreb, Croatia. The main clinical characteristics of this patient cohort are reported in Online Supplementary Table S2. A detailed description of preparation of slides can be found in the Online Supplementary Appendix.

\section{Proliferation assays}

Full details can be found in the Online Supplementary Appendix.

\section{Intracytoplasmic staining for cytokine production}

PBMC were activated for four days with anti-CD3 and -CD28 $\mathrm{mAbs}$, as detailed above, with or without recombinant soluble PD-L1 (sPD-L1, $10 \mu \mathrm{g} / \mathrm{mL}, \mathrm{R} \& D$ Systems). Brefeldin A (eBioscience) was added during the final four hours of culture. Cytokines were measured by intracytoplasmatic staining with anti-IL4-FITC and anti-IFN- $\gamma$-PE Abs after gating on $\mathrm{CD}^{+}$or $\mathrm{CD}^{+}$ subsets, respectively.

\section{ELISpot assay}

The frequency of IFN- $\gamma$-secreting T lymphocytes in cultures was assessed in an ELISpot assay, as previously described. ${ }^{16}$ Full details are to be found in the Online Supplementary Appendix.

\section{Statistical analyses}

Statistical analyses were carried out with GraphPad version 5 (GraphPad Software Inc, La Jolla, CA, USA) and the data are presented as box plots where the box represents the $25^{\text {th }}$ (bottom) and $75^{\text {th }}$ (top) percentiles; the band inside the box is the median. The whiskers represent the minimum and maximum of all data. In the text, data are presented as median \pm standard error.

Continuous variables were compared by Mann-Whitney test. Wilcoxon's matched-pairs signed rank test was used for paired continuous variables. Data were analyzed with Prism software version 5.0 (GraphPad Software).

\section{Results}

\section{$C D 4^{+}$and $C D 8^{+} T$ lymphocytes from CLL patients show increased PD-1 expression as compared to controls with a comparable age}

PD-1 surface expression was tested by flow cytometry in T lymphocytes from CLL patients $(n=117)$ and compared to controls $(n=33)$. The patients were either untreated or had received the last course of chemotherapy more than three months previously. Three patients $(8 \%)$ had received anti-CD52-containing regimen (Online Supplementary Table S1). By applying a sequential gating strategy, based on morphological parameters and multicolor flow cytometry (Online Supplementary Figure S1), PD1 was significantly increased in the $\mathrm{CD}^{+}$T-cell subset from CLL patients, as compared to controls (Figure 1A). Similar results were obtained when the $\mathrm{CD} 8^{+}$subset was analyzed (Figure 1B). CLL patients displayed high variability in both T-cell subsets, while PD-1 was homogeneously expressed in T lymphocytes from all control samples tested. Moreover, a fraction of CLL lymphocytes, identified on the basis of CD19/CD5 surface expression, was PD-1 (Figure 1C), confirming previous observations. ${ }^{17}$ On the contrary, circulating $\mathrm{B}$ lymphocytes from controls were mostly PD-1- (Figure 1C).

The PD-L1 ligand was found to be restricted to B lymphocytes in CLL and control samples; however, CLL B lymphocytes expressed significantly more PD-L1 when 
A

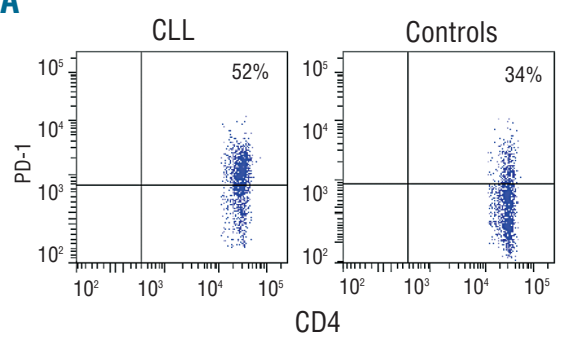

C
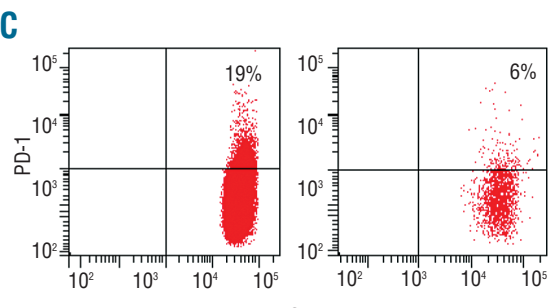

CD19
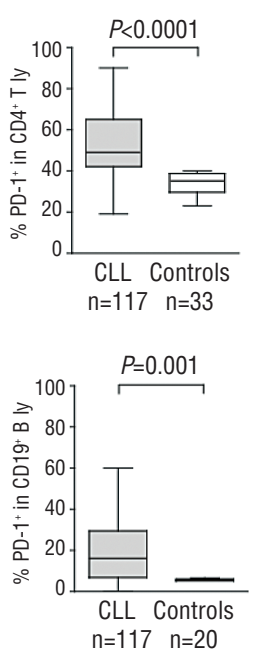

B
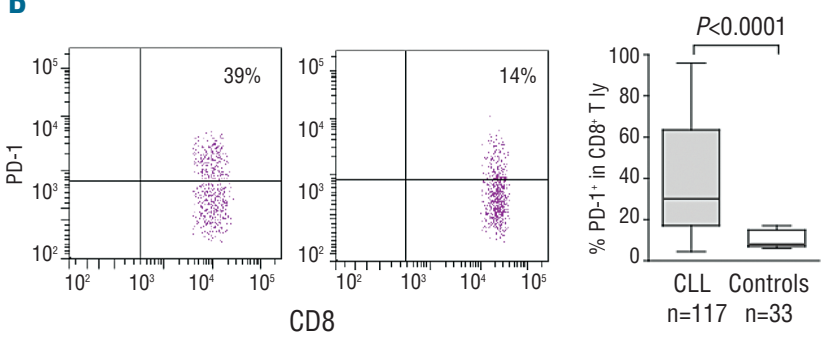

D
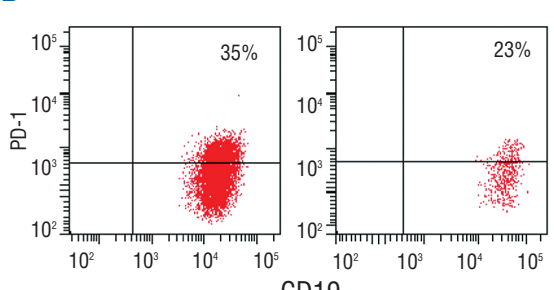

Figure 1. Comparative analysis of the surface expression of PD-1 and its ligand PD-L1 in distinct lymphocyte subsets from CLL patients and from age- and sex-matched donors. Representative dot plots showing surface expression of PD-1 in CD4 ${ }^{+}(A), \mathrm{CD}^{+}(\mathrm{B})$ and $\mathrm{CD}^{-19} \mathrm{9}^{+}$lymphocytes (C) from CLL patients (left side) or age- and sex-matched donors (right side). The expression of the PD-L1 ligand in the CD19 ${ }^{+}$subset is shown in (D). Cumulative data from $117 \mathrm{CLL}$ patients (gray bars) and 33 controls (open bars) are reported in the box plots on the right.

compared to B lymphocytes cells from controls (Figure 1D).

\section{T lymphocytes from CLL patients show a marked increase in terminally differentiated populations}

$\mathrm{PD}-1$ in human circulating $\mathrm{CD} 4^{+} \mathrm{T}$ cells marks chronically activated effector memory cells. ${ }^{18-20}$ On the contrary, $\mathrm{PD}$ 1 is classically considered a marker of cell exhaustion in the CD8 subset. ${ }^{21}$ Thus, the finding of an increased expression of PD-1 in T lymphocytes from CLL patients suggests a relative enrichment in terminally differentiated cells as compared to controls with a similar age.

This hypothesis was tested by analyzing T-cell subpopulations in the same cohorts as above. To do so, $\mathrm{CD}^{+}$and CD8 ${ }^{+}$subset cells were stained with CD45RA and CCR7 in order to quantify the relative number of naïve, central memory, effector memory and terminally differentiated cell $^{22}$ (Figure 2A). The latter population is virtually absent in the $\mathrm{CD}^{+}$subset under physiological conditions, but becomes apparent during chronic activation. ${ }^{23,24}$ Representative profiles from a CLL patient and a control are reported in Figure 2A. Cumulative data indicated marked differences in the composition of both $\mathrm{CD}^{+}$and $\mathrm{CD} 8^{+} \mathrm{T}$-cell subsets (Figure $2 \mathrm{~B}$ ). The most significant differences in the $\mathrm{CD} 4^{+}$compartment concerned the decrease of naive cells and the relative increase of terminally differentiated populations. Similar results were found in the $\mathrm{CD} 8^{+}$compartment, with a decrease of central memory cells and a concomitant increase in terminally differentiated cells. When determining PD-1 expression in the different subsets we unexpectedly found that the molecule was expressed by each subpopulation, including naïve cells. However, it was clear that $T$ lymphocytes from the 8 examined CLL patients displayed significantly higher levels of PD-1 in naive, central and effector memory subsets when compared to the normal counterpart $(n=5)$. Terminally differentiated cells did not reach statistical sig- nificance (Online Supplementary Figure S2). Increased PD-1 expression was also noted in naive and central memory CD8+ CLL T lymphocytes. No differences were observed when comparing effector memory or terminally differentiated cells (Online Supplementary Figure S2).

\section{The increase in effector memory CD4 ${ }^{+}$and terminally differentiated CD8 ${ }^{+}$lymphocytes parallels disease aggressiveness}

The heterogeneity in T-cell subset composition might indicate that the degree of $\mathrm{T}$-cell exhaustion varies according to the stage and aggressiveness of the leukemia. For this reason, the patient cohort was divided on the basis of clinical and molecular markers of disease burden and aggressiveness. Patients with a more advanced stage of disease, as well as patients who required treatment, were characterized by higher levels of circulating $\mathrm{CD}^{+}$effector memory cells (Figure 2C). The same results were obtained when considering terminally differentiated $\mathrm{CD} 8^{+}$cells (Figure 2D).

In line with these results, the percentage of effector memory $\mathrm{CD}^{+}$and terminally differentiated $\mathrm{CD} 8^{+}$lymphocytes was significantly lower in patients characterized by favorable molecular markers, such as the presence of mutations in the IgHV genes (Figure 2C) and the absence of deleterious chromosomal deletions than in the counterpart (Figure 2D).

\section{PD-1 and PD-L1 are significantly up-modulated in proliferating $T$ lymphocytes and CLL cells}

We then asked whether PD-1/PD-L1 expression could be modulated during cell activation. T lymphocytes from CLL patients were activated using mitogenic anti$\mathrm{CD} 3 / \mathrm{CD} 28 \mathrm{mAbs}$ and stained using CFSE. Proliferating $\mathrm{CD}^{+}$and the $\mathrm{CD} 8^{+}$subsets were identified after applying a morphological (FCS vs. SSC) gate, followed by staining with anti-CD4 or -CD8 (Figure 3A). PD-1 expression 
increased significantly in proliferating cells. The increase was predominant in the $\mathrm{CFSE}^{+}$proliferating compartment, with intensity varying in function of the number of proliferation cycles (Figure 3B). PD-1 increase following activation could also be observed in T cells from controls, as previously described ${ }^{25}$ (Figure $\left.3 \mathrm{~B}\right)$. However, the levels of PD-
1 expression in activated $\mathrm{CD}^{+}$and $\mathrm{CD}^{+} \mathrm{T}$ lymphocytes from CLL patients were significantly higher than in controls (Figure 3B).

Expression of the PD-L1 ligand increased sharply when purified CLL cells were induced to proliferate by culturing with immunostimulatory $\mathrm{CpG}$ nucleotides and IL-2. ${ }^{26}$
A

CLL

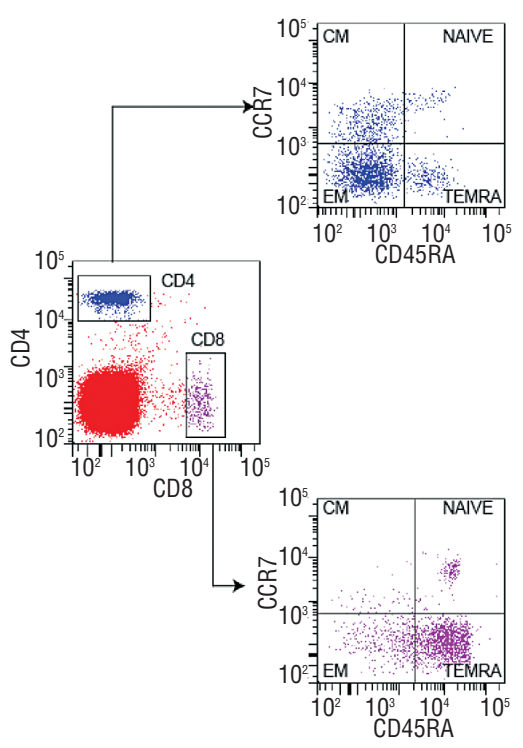

B

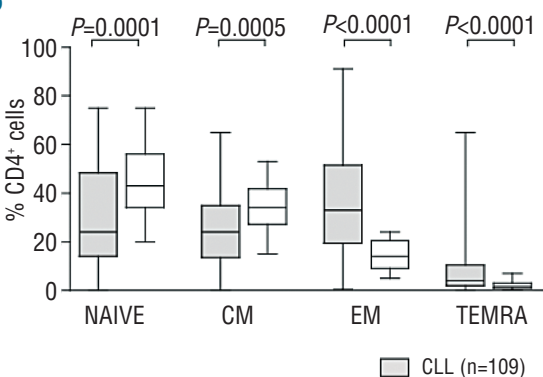

C

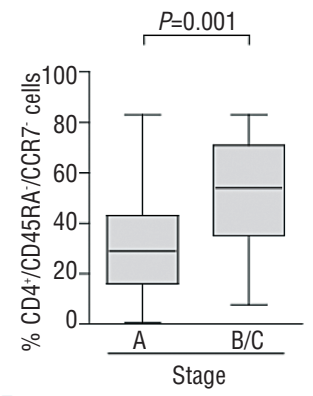

D
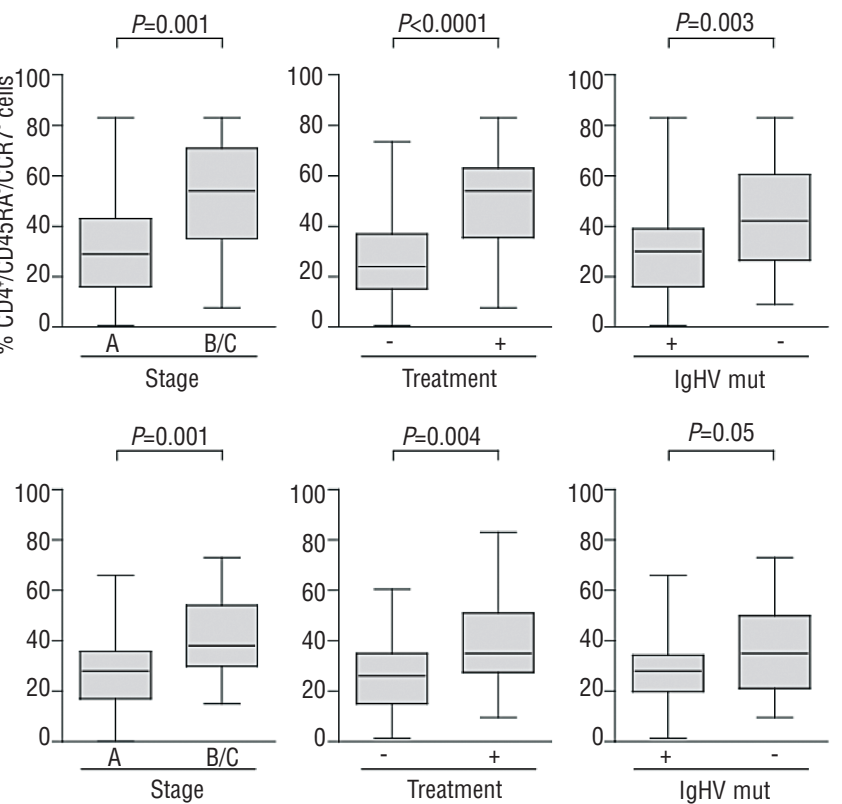
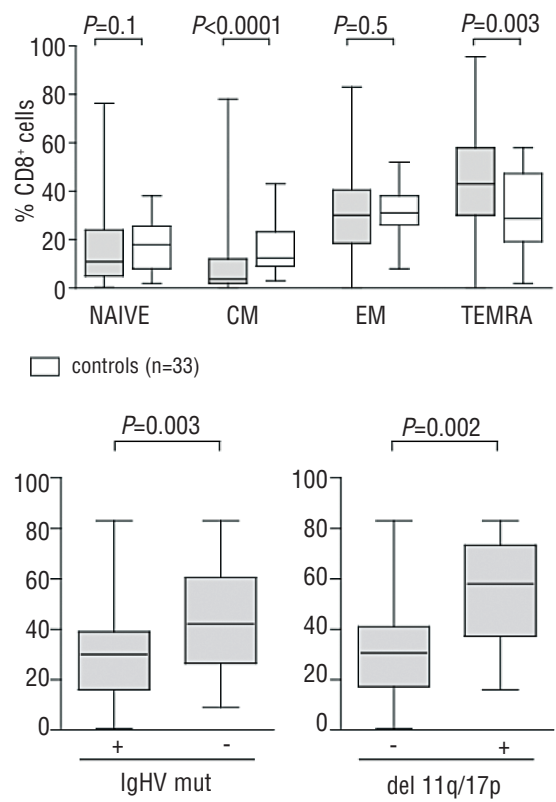

$\square$ controls (n=33)

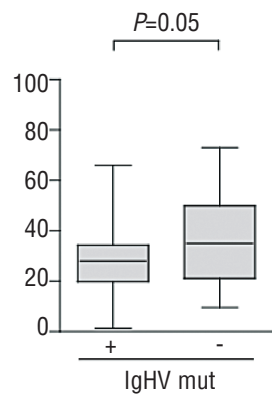

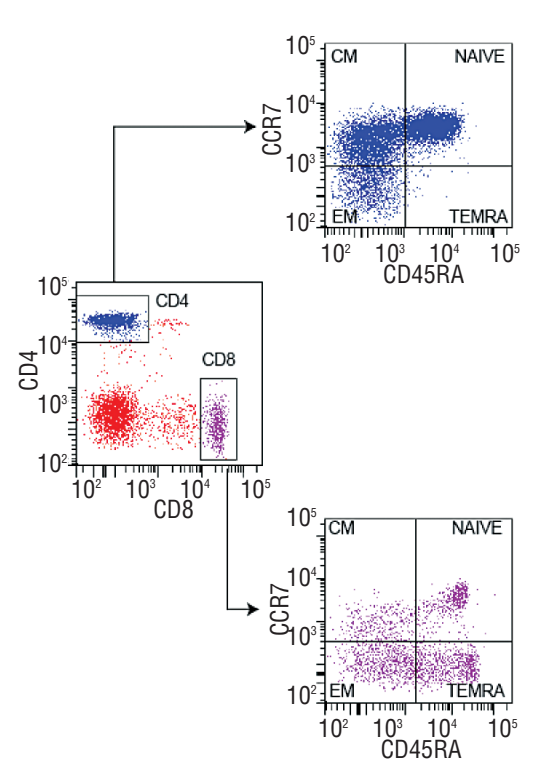

Controls

Figure 2. Comparative analysis of Tlymphocyte subpopulations in $\mathrm{CLL}$ patients and in age- and sex-matched donors. (A) Representative dot plots showing flow cytometric analysis of PBMC from CLL patients (left) or controls (right) after staining for CD4, CD8, CD45RA and CCR7. A first gate was applied to identify $\mathrm{CD4}^{+}$(blue) or $\mathrm{CD8}^{+}$(purple) subsets. The combination of CD45RA and CCR7 resulted in the identification of naïve $\left(\mathrm{CD}^{2} 5 \mathrm{RA}^{+} / \mathrm{CCR}^{+}\right)$, central memory (CD45RA /CCR7 $7^{+}$), effector memory (CD45RA /CCR7) and terminally differentiated (CD45RA ${ }^{+}$CCR7) subpopulation. (B) Cumulative data showing the distribution of $\mathrm{CD}^{+}$(left graph) or $\mathrm{CD}^{+}$(right graph) lymphocyte subpopulations in 109 CLL patients (gray box plots) and 30 age-and sex-matched controls (open bars). (C) and (D) Box plots showing cumulative distribution of $\mathrm{CD}^{+} / \mathrm{CD}^{-} 5 \mathrm{RA}^{-} / \mathrm{CCR} 7^{-}$effector memory $\mathrm{CD}^{+}$cells and of $\mathrm{CD}^{+} / \mathrm{CD}^{2} 5 \mathrm{RA}^{+} / \mathrm{CCR} 7-$ terminally differentiated $\mathrm{CDB}^{+}$cells, respectively, in patient subsets divided according to clinical markers of disease burden (Binet stage or treatment requirement) or to molecular markers of disease aggressiveness (presence or absence of mutations in the IgHV genes and of deletions in chromosomes $11 q$ and $17 p$ ). 
Proliferating CLL cells acquired PD-L1 surface expression, with the majority of the clone becoming strongly positive (Figure 3C). PD-L1 upregulation in B lymphocytes from controls activated in the presence of the same signals was only marginal and markedly lower than that observed in proliferating CLL cells (Figure $3 \mathrm{C}$ ). These results suggest that the PD-1/PD-L1 axis is maximally active in an activated environment.

\section{$P D-1^{+} T$ lymphocytes are in close contact} with PD-L1 CLL cells in the PC

The proliferative CLL compartment is predominantly hosted within the LN. For this reason, PD-1 and PD-L1 expression patterns were tested by immunohistochemistry and confocal microscopy in LN specimens obtained from 20 patients with a confirmed diagnosis of CLL. All samples displayed an almost complete effacement of the

A
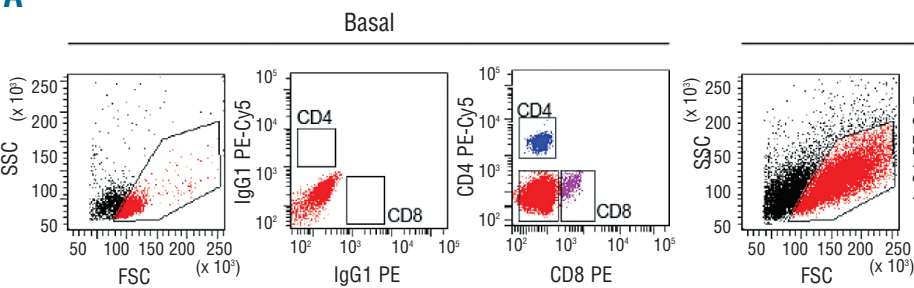

Activated

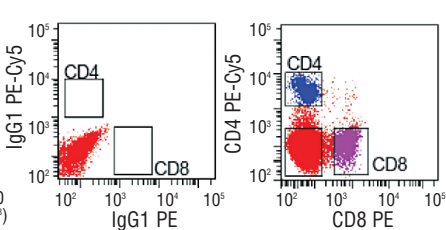

B
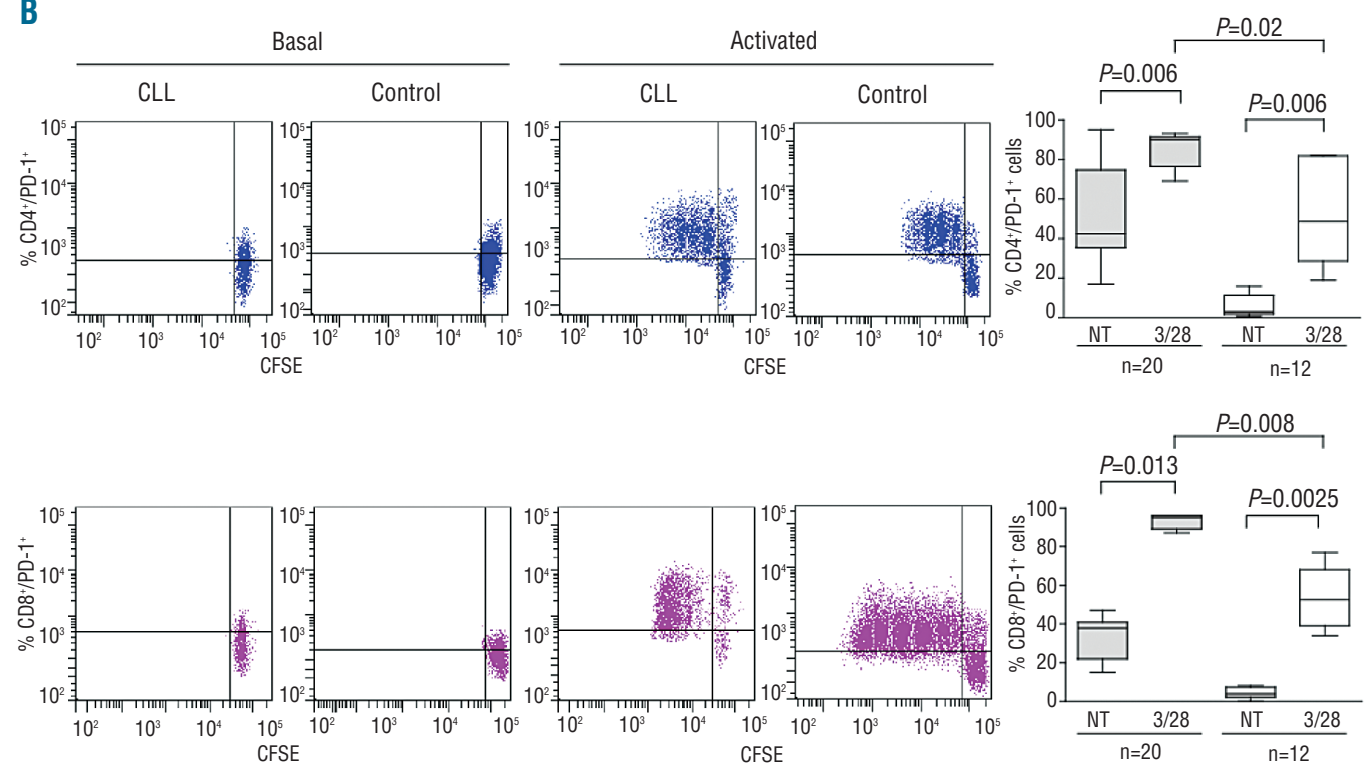

C
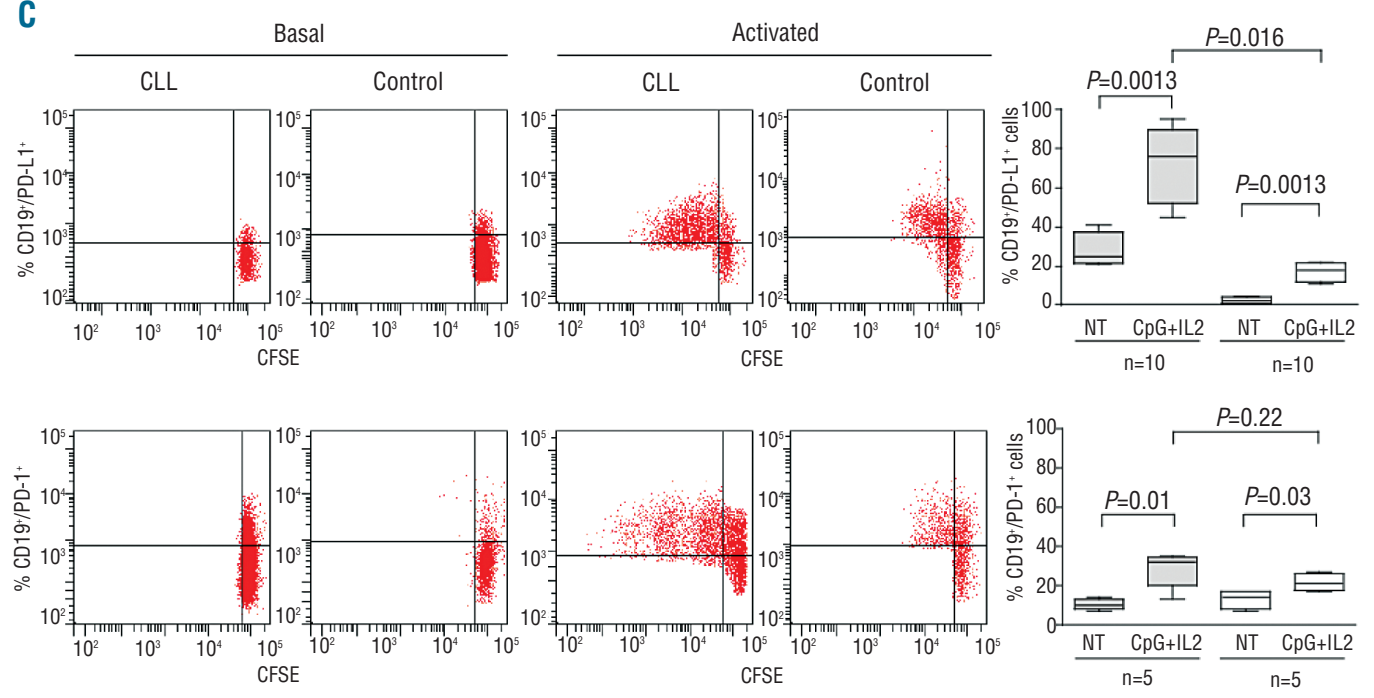

$\square$ CLL $\square$ Control

Figure 3. Expression of the PD-1/PD-L1 pair in proliferating $\mathrm{T}$ lymphocytes and CLL cells. CFSE-labeled PBMC preparations from CLL patients or age- and sex-matched controls were treated with mitogenic antiCD3 and anti-CD28 antibodies to trigger Tcell proliferation or with a combination of $\mathrm{CpG}$ oligonucleotides and recombinant human IL-2 to induce B-cell proliferation. Morphological gating based on FCS and SSC parameters and subsequent staining with specific antibodies allowed to identify $\mathrm{CD}^{+}$(blue), $\mathrm{CD}^{+}$(purple) and $\mathrm{CD}^{+}{ }^{+}$(red) subsets (A) Representative do plots show PD-1 and PD-L1 expression in proliferating $\mathrm{CD}^{+}$, $\mathrm{CD}^{+}$(B) and $\mathrm{CD}^{+} 9^{+}$ (C) subpopulations. Cumulative data from CLL patients (gray bars) and age- and sex-matched controls (open bars) are shown in the box plots on the right. 
normal LN architecture by CLL cells, along with evidence of morphologically distinct PC, with areas rich in prolymphocytes and para-immunoblasts. PD-1+ $1^{+}$cells appeared more concentrated in the paler areas classically associated with the PC (Figure 4A), as also confirmed after Ki-67 staining (data not shown). Quantification of staining intensity showed that PD-1 was expressed at significantly higher levels in the PC as compared to the other areas of the specimen (Figure 4B). High magnification images indicated that PD-1 staining was limited to surface membranes and associated with a distinct cell subset, overlapping almost completely with the T-cell infiltrate, as identified by CD2 staining in immunofluorescence experiments (Figure 4C). Co-staining with an anti-CD4 $\mathrm{mAb}$ indicated that the majority of these $\mathrm{T}$ lymphocytes are $\mathrm{CD}^{+}{ }^{+}$(Online Supplementary Figure S3). Reactive LN samples with clearly identifiable and conserved follicles were characterized by a subset of predominantly small lymphocytes in the germinal centers showing intense staining for PD-1, in line with previous data. ${ }^{27,28} \mathrm{PD}-1$ staining was also apparent in the interfollicular areas, predominantly containing $\mathrm{T}$ lymphocytes (Figure 4D). Also in the case of reactive $\mathrm{LN}$, immunofluorescence confirmed that $\mathrm{PD}-1^{+}$cells overlapped almost completely with the $\mathrm{CD}^{+}$subset (Figure $4 \mathrm{E})$ and were mostly $\mathrm{CD}^{+}$(Online Supplementary Figure S3).

PD-L1 expression in LN samples from CLL patients was quite heterogeneous, with some samples (9 of 20) showing a diffuse pattern, while others (10 of 20) displayed a patchier reactivity and one sample was completely negative. (Figure 5A). In any case, the strongest intensity was associated to the $\mathrm{PC}$, as shown after quantification of $\mathrm{PD}$ L1 staining (Figure 5B). The PD-L1 staining pattern did not show any clear-cut association with clinical parameters, including disease stage or treatment, with the limits of the cohort examined (Online Supplementary Table S2). Immunofluorescence confirmed that PD-L1+ elements were consistently CD23+ (Figure 5C), with the exception
A

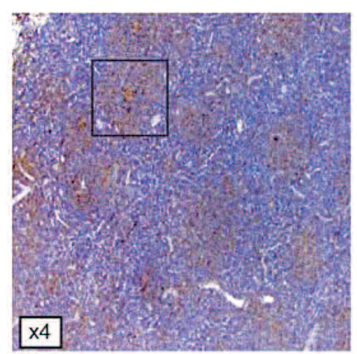

C

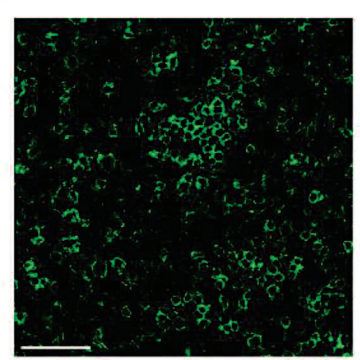

D

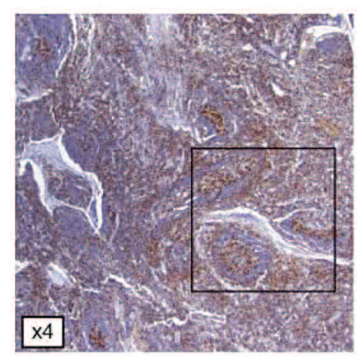

E

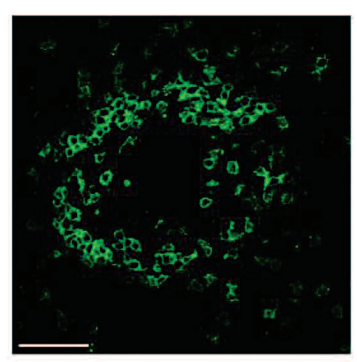

$\mathrm{PD}-1$

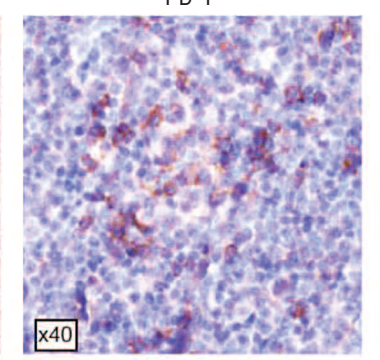

Green: PD-1; Red: CD2

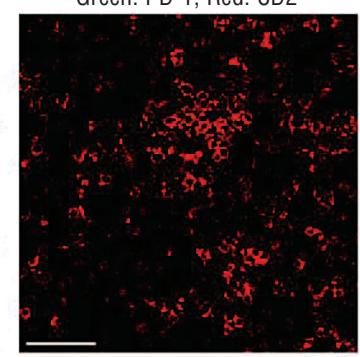

PD-1

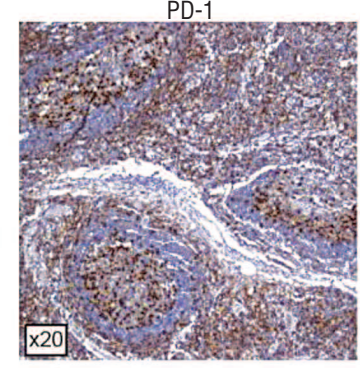

Green: PD-1; Red: CD2

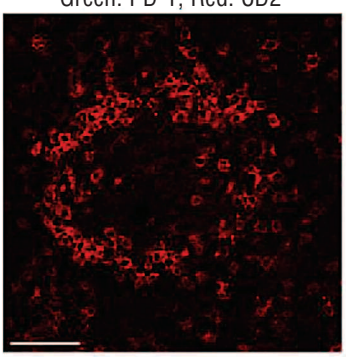

B
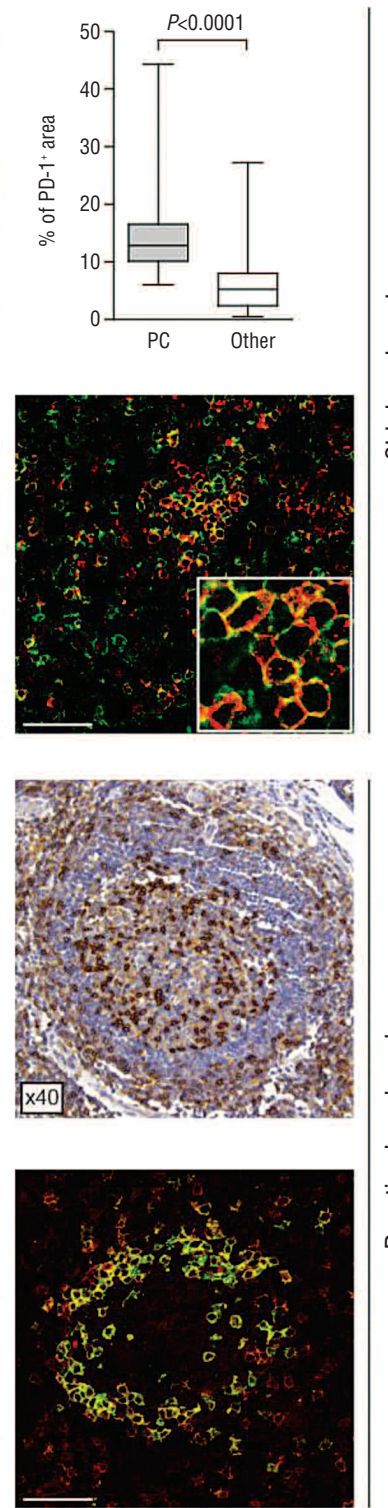

Figure 4. Immunohistochemistry and immunofluorescence analysis of PD1 expression on CLL and reactive $\mathrm{LN}$. (A) Anti-PD-1 immunostaining of a representative LN tissue section using a goat HRP-polymer detection system and 3,3'-diaminobenzidine (brown signal). Low magnification shows PD-1 expression around the paler areas corresponding to the proliferation centers. Original magnification 4x and 40x. (B) Quantification of staining intensity was performed by comparing the percentage of PD- $1^{+}$ areas in 20x magnification images of PC (shaded box plot) to other (open box plot) parts of the same slide. Analyses were performed using 4 different samples and at least 6 independent images of PC vs. 6 of other areas per sample. (C) Double staining of a representative CLL LN tissue section with anti-PD-1 (green) and anti-CD2 (red). Merged panel shows that the majority of $\mathrm{PD}-1^{+}$cells are CD2 ${ }^{+}$T lymphocytes. Original magnification 63x. Scale bar represents 50 $\mu \mathrm{m}$. (D) Anti-PD-1 immunostaining of a representative reactive LN tissue section. (E) Double staining of a representative germinal center from a reactive $L N$ tissue section with antiPD-1 (green) and anti-CD2 (red). Original magnification 63x. Scale bar represents $\quad 50 \quad \mu \mathrm{m}$ Immunohistochemical images were analyzed using a Leica DMI 3000 B optical microscope (Leica Microsystesms), equipped with a DCF 310 FX digital camera and LAS V3.8 software. Immunofluorescence analysis was performed using a TCS SP5 laser scanning confocal microscope (Leica Microsystems) with an oil immersion $63 x / 1.4$ objective lenses, images were acquired with the LAS AF software and processed with Adobe Photoshop (Adobe Systems, San Jose, CA, USA). 
of small vessels, which were confirmed as weakly PD-L1 ${ }^{28}$ (Figure 5C).

Simultaneous staining for PD-1, PD-L1 and CD3 in LN samples from CLL patients indicate that PD-L1+ leukemic lymphocytes are preferentially located in close proximity to PD-1+ T lymphocytes (Figure 5D). This conclusion was confirmed by quantifying relative fluorescence intensity of PD-L1 in $\mathrm{CD}^{\text {high }}$ vs. $\mathrm{CD}^{\text {low }}$ areas (Figure $5 \mathrm{E}$ ).

The expression of PD-L1 in reactive $\mathrm{LN}$ samples was confined to the endothelial cells lining the vessels (Figure $5 \mathrm{~F}$ ), as also confirmed by immunofluorescence (Online Supplementary Figure S4).

In conclusion, data from tissue sections indicate that the PC of CLL LN hosts CD4 ${ }^{+} \mathrm{T}$ lymphocytes expressing PD1 and CLL cells expressing the PD-L1 ligand, suggesting a direct contribution of the PD-1/PD-L1 axis in the intense crosstalk occurring between the two cell populations in this environment.

\section{PD-1/PD-L1 crosstalk contributes to suppressing IFN- $\gamma$ production in $\mathrm{CDB}^{+} \mathrm{T}$ lymphocytes}

Multiple reports indicate that T lymphocytes from CLL patients are partially dysfunctional, with a Th2 skewing. ${ }^{29,30}$ Our results obtained with intracytoplasmic staining confirmed that $\mathrm{CD}^{+} \mathrm{T}$ lymphocytes from CLL patients $(n=12)$ produce significantly more IL-4 than those obtained from appropriate controls when activated in the presence of mitogenic anti-CD3/CD28 mAbs, (Figure 6A and $\mathrm{B})$. On the contrary, $\mathrm{CD}^{+} \mathrm{T}$ lymphocytes from CLL patients produced significantly lower amounts of IFN- $\gamma$ as compared to controls (Figure 6A and B). No significant differences between CLL and controls were observed in terms of proliferative responses (data not shown).

We then asked whether the PD-1/PD-L1 axis can modulate cytokine secretion by different T-cell subsets, contributing to the Th2 skewing that characterizes T-cell responses in CLL. To do so, T lymphocytes were treated
A

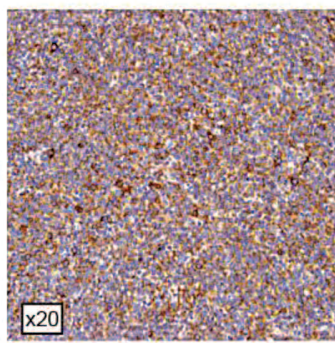

C

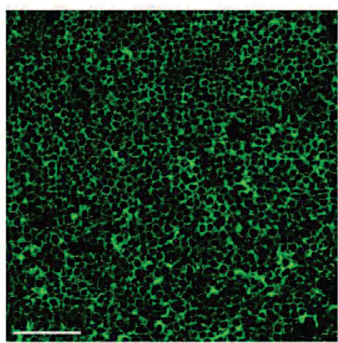

D

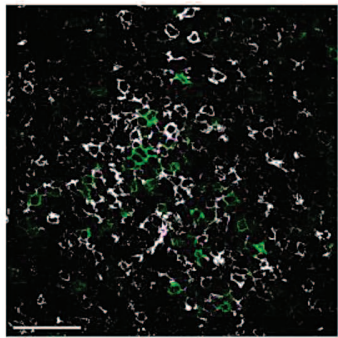

$\mathrm{E}$

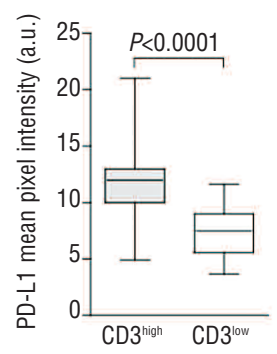

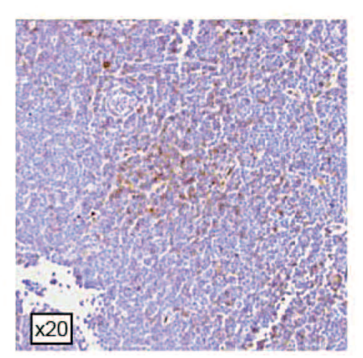

Green: CD23; Red: PD-L1

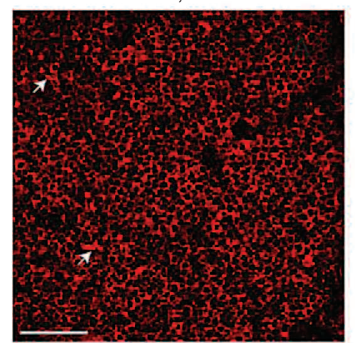

Green: PD-1; White: PD-L1; Red: CD3

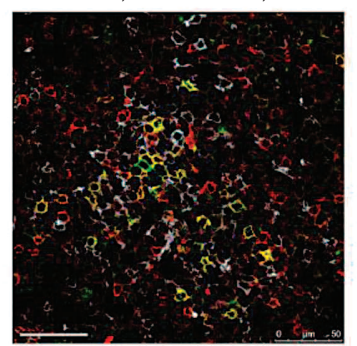

PD-L1

F

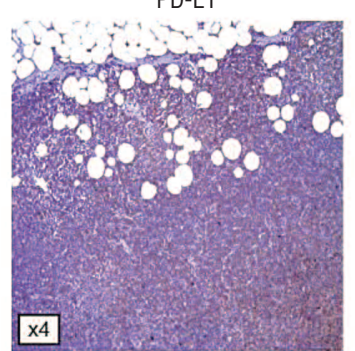

B
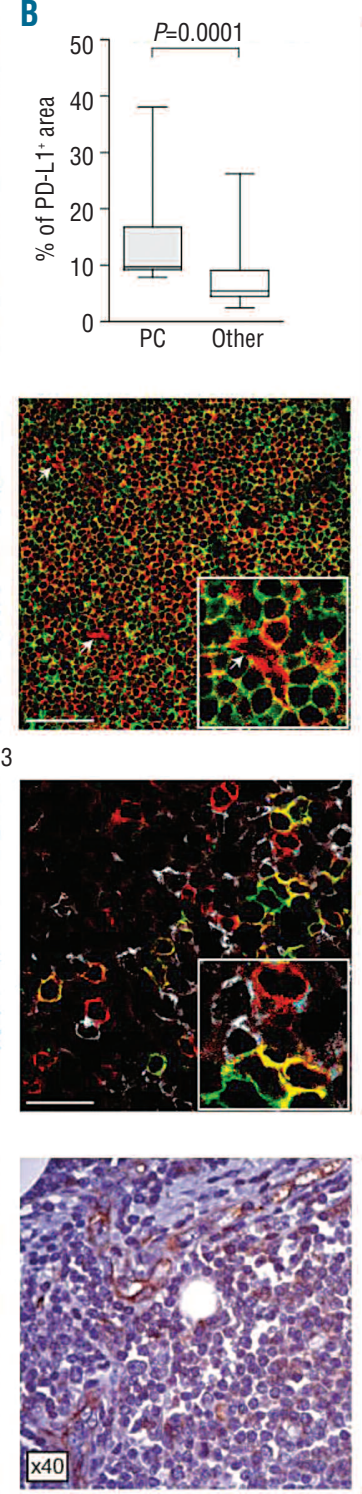

Figure 5. PD-L1 expressing CLL cells are in close contact with PD-1 ${ }^{+} \mathrm{T}$ lymphocytes. (A) Anti-PD-L1 immunostaining of two representative CLL LN tissue sections, displaying a diffuse or a patchy reactivity. Original magnification 20x. (B) Quantification of PD-L1 ${ }^{+}$areas showed that the highest expression is associated to the PC. Analyses were performed using 4 different samples and at least 6 independent images of PC vs. 6 of other areas per sample. (C) Double staining of a representative CLL LN section with anti-CD23 (green) and anti-PD-L1 (red) confirms that PD-L1 expressing cells are $\mathrm{CD}^{+}{ }^{+} \mathrm{CLL} B$ lymphocytes. The white arrows indicate the vessels, which are stained positive for PD-L1. Original magnification $63 x$. Scale bar represents 50 um. (D) Double staining (left panel) for PD-1 (green) and PD-L1 (white) and triple staining (right panels) for PD-1 (green), PD-L1 (white) and CD3 (red) of a representative CLL LN tissue section. Original magnification 63x, zoom factor of 2 for image on the right. Scale bars represent $50 \mu \mathrm{m}$ (left panels) and 25 $\mu \mathrm{m}$ (right panel). (E) Comparative analysis of PD-L1 pixel intensity (arbitrary units, a.u.) in CD3high vs. CD3low areas from different LN sections indicates significantly higher intensity of expression in close proximity of PD-1 $1^{+} / \mathrm{CD}^{+} \mathrm{T}$ lymphocytes. For cumulative analysis 10 randomly chosen fields from 3 different samples were counted. (F) Anti-PD-L1 immunostaining of a representative reactive $\mathrm{LN}$ tissue section. In contro samples the PD-L1 expression is confined to endothelial cells. Immunohistochemical images were analyzed using a Leica DMI 3000 B optical microscope (Leica Microsystesms), equipped with a DCF 310 FX digital camera and LAS V3.8 software. Immunofluorescence was analyzed using a TCS SP5 laser scanning confocal microscope (Leica Microsystems) with an oil immersion $63 x / 1.4$ objective lenses, images were acquired with the LAS AF software and processed with Adobe Photoshop (Adobe Systems). Pixel intensities were calculated with the ImageJ software. 
with a recombinant soluble form of the PD-L1 ligand to trigger $\mathrm{PD}-1^{+}$cells. The production of IFN- $\gamma$ and IL-4 were selected as indicators of the quality and quantity of the signals induced and were measured by intracytoplasmic staining. Results indicate that $\mathrm{T}$ lymphocytes from CLL patients responded to PD-1 engagement by significantly decreasing IL-4 and IFN- $\gamma$ production in the CD4 $4^{+}$ and $\mathrm{CD}^{+}$subsets, respectively (Figure $6 \mathrm{~B}$ ). Similar results were obtained using control $\mathrm{T}$ lymphocytes (Figure 6B). A direct measure of IFN- $\gamma$ secretion by CLL T lymphocytes using an ELISpot assay confirmed a significant decrease in the presence of soluble PD-L1. Conversely, treatment with a blocking anti-PD-L1 anti- body led to a significant increase in IFN- $\gamma$ secretion by CLL T lymphocytes. The stimulatory effect was even more evident when anti-PD-L1 antibody was combined with an anti-PD-1 reagent (Figure 6C). The same experiments performed using $\mathrm{T}$ lymphocytes from age- and sex-matched donors yielded significant modulation of IFN- $\gamma$ production only in the presence of a combination of blocking anti-PD-1 and anti-PD-L1 antibodies, likely suggesting that this axis is less active in normal $\mathrm{T}$ cells (Figure 6C). Thus, the engagement of PD-1 and PDL-1 among host $\mathrm{T}$ cells and CLL cells within the PC may skew the immune responses and contribute to a dysregulated Th2 response.
A
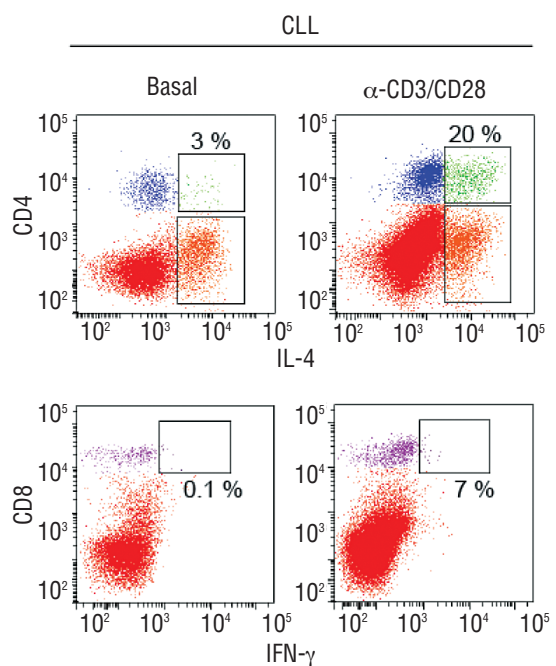

B

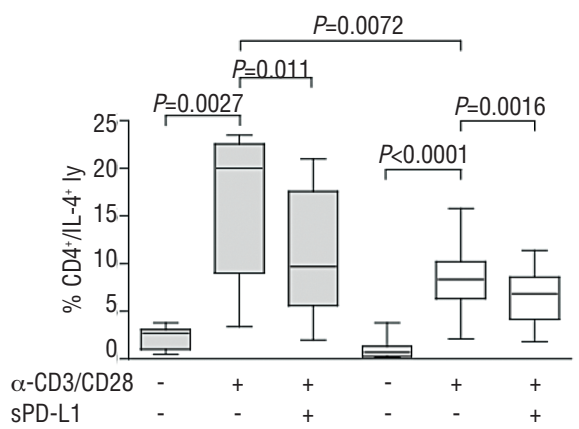

CLL $(n=12)$

C

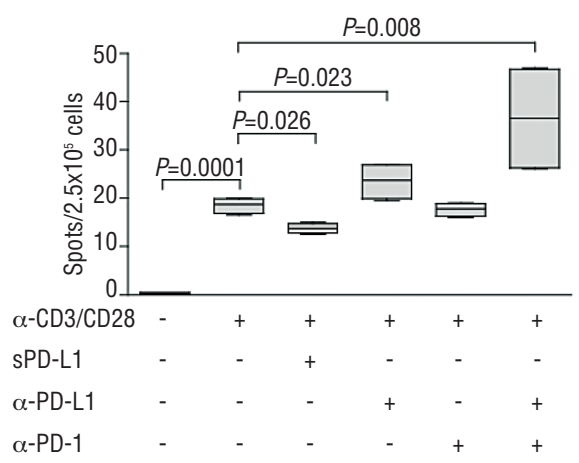

$\square \operatorname{CLL}(\mathrm{n}=4)$
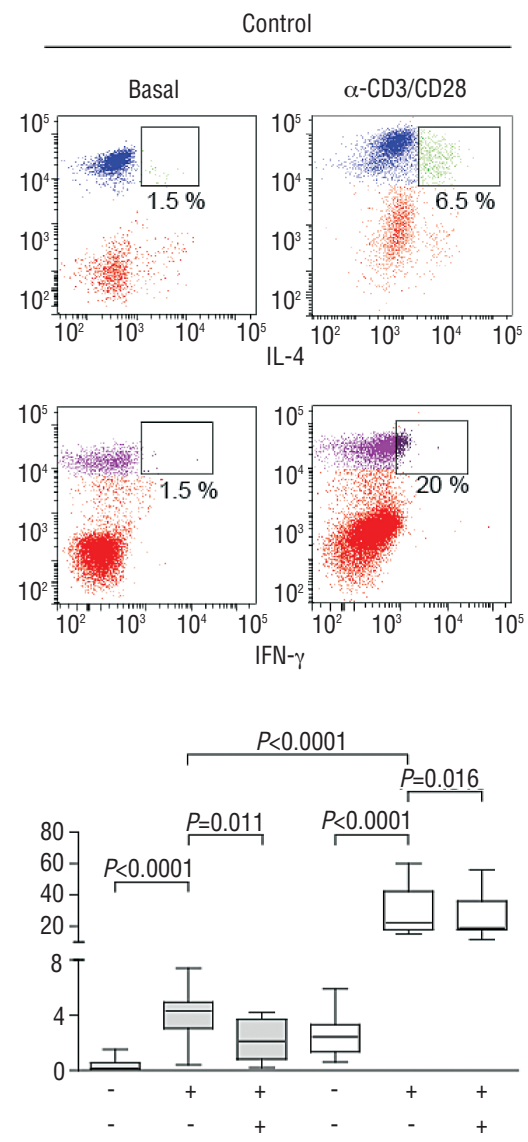

Controls ( $\mathrm{n}=12$ )

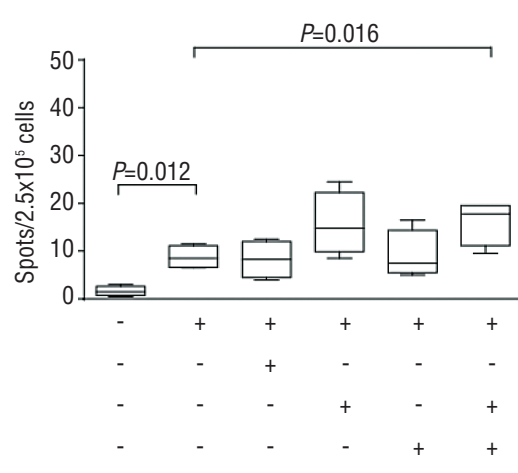

Controls ( $\mathrm{n}=4$ )
Figure 6. IL-4 $4^{+}$and IFN- $\gamma^{+}$production by $\mathrm{CD}^{+}$and $\mathrm{CD}^{+} \mathrm{T}^{-}$ lymphocytes blocking PD1/PD-L1 axis. (A) Representative dot plots showing intracytoplasmatic IL4 staining in $\mathrm{CD}^{+}$(upper panel) and IFN- $\gamma$ staining in $\mathrm{CD8}^{+}$(lower panel) T lymphocytes from CLL patients (left side) or matched donors (right side). Cells were tested in basal conditions or after culture with mitogenic antiCD3/CD28 mAbs. (B) Cumulative data from $12 \mathrm{CLL}$ patients (gray box plots) and 12 age- and sex-matched controls (open box plots) are shown in the box plots in basal vs. mitogenic conditions. T lymphocytes were otherwise treated with a recombinant soluble form of the PD-L1 ligand. (C) Cumulative data from ELISpot experiments showing IFN- $\gamma$ production by 4 different CLL (gray box plots) or 4 matched controls (open box plots) after activation using anti-CD3 and anti-CD28 antibodies in combination with soluble PD-L1, anti-PD-1 and anti-PD-L1 antibodies. 


\section{Discussion}

Immunoediting is a phenomenon by which cancer cells alter their phenotype in order to escape attack from the immune system. Even if this is more effective in the early stages of tumorigenesis, there is ample evidence to suggest that during progression tumor cells continue to exploit a wide variety of subversive processes aimed at maintaining their own survival. ${ }^{31}$ At least in solid tumors, one such process relies on the overexpression of the surface molecule PD-L1, which can engage the co-receptor PD-1 on surrounding $\mathrm{T}$ lymphocytes and inhibit T-cell responses, consequently permitting cancer progression and diffusion. ${ }^{32,33}$ Targeting this receptor-ligand pair using specific antibody therapy leads to impressive clinical responses in patients with solid tumors of different origins. ${ }^{34,35}$

On the contrary, data and information on the expression and function of PD-1 and its ligands in normal and neoplastic lymphoid tissues is more limited. In normal human tonsils, PD-1 appears to be expressed by follicular T-helper cells localized in the light zone of germinal centers (GCs). ${ }^{38}$ Germinal center B cells, at least in the mouse, are PD-L1+ and PD-L2': PD-1/PD-L1 interactions control the full development of follicular T-helper cells which regulate the generation of long-lived plasma cells. ${ }^{36}$ In the neoplastic context, PD-1 is reported as expressed by tumor infiltrating, as well as circulating, T lymphocytes in Hodgkin's and non-Hodgkin's lymphomas and in adult T-cell leukemia. ${ }^{27,37,38}$ Furthermore, PD-1+ $1^{+}$helper $\mathrm{T}$ cells were identified as an independent prognostic risk factor for decreased overall survival in follicular lymphoma. ${ }^{39}$ Expression of PD-1 on the neoplastic counterpart was reported in a limited number of diffuse large B-cell lymphomas and in a more significant number of small lymphocytic lymphomas. A fraction of circulating CLL cells is also reported as PD-1 ${ }^{+}$(see Grzywnowicz et al. ${ }^{17}$ and Li et al. ${ }^{18}$ and confirmed in this work) even if the prognostic and functional implications remain unknown.

While comparing PD-1 expression by T lymphocytes in a cohort of 117 CLL patients to normal donors matched for age and male/female ratio, we observed a significant increase in the percentage of positive cells in the $\mathrm{CD} 4^{+}$and the $\mathrm{CD} 8^{+}$subsets. This finding could be interpreted considering the altered composition of circulating $\mathrm{T}$ lymphocytes, marked by decreased naïve $\mathrm{T}$ cells and a concomitant increase of more differentiated $T$ subsets. This imbalance is sharpened during disease progression: the number of effector memory $\mathrm{CD} 4^{+}$and terminally differentiated $\mathrm{CD}^{+}$lymphocytes correlates with clinical and molecular markers of disease burden and aggressiveness. However, analysis of PD-1 expression in different T-cell populations, as divided on the basis of CD45RA and CCR7 expression, indicated high levels in all subsets, including naive cells. This somewhat unexpected result (in the literature, PD-1 is classically associated to a memory phenotype) possibly reflects an age-related effect. In any case, PD-1 expression levels were constantly higher in all T-cell subpopulations, both of CD4 and of CD8 origin, purified from CLL patients than controls. In the limited cohort of patients studied, PD-1 expression levels were not significantly associated to a specific subgroup of patients, at least as defined by stage of disease, treatment requirements or expression of molecular or cytogenetic markers of unfavorable prognosis. This negative finding could be explained considering that cell activation can rapidly mod- ulate PD-1 expression on T lymphocytes. ${ }^{21}$ The results of this work indicate that PD-1 expressed by T lymphocytes from CLL patients robustly increases when the cells are activated in an antigen-specific way. An alternative explanation is that the relevant $\mathrm{PD}-1^{+}$population is the residential one, only marginally and indirectly represented within the peripheral pool. In line with this view, immunohistochemical analyses of PD-1 expression within the CLL LN showed the presence of an intensely positive population of T lymphocytes. These cells were present at higher density within the PC and appeared to be in close contact with $\mathrm{CD}_{2} 3^{+}$leukemic elements. Immunofluorescence studies further indicated that the vast majority of $\mathrm{PD}-1^{+} \mathrm{T}$ cells belong to the CD4 lineage, with no detectable expression being associated to $\mathrm{B}$ cells in this district. On the contrary, PD-L1 staining in reactive LN samples was mostly associated with endothelial cells lining small vessels. ${ }^{40,41}$ In the same samples, follicular T-helper cells of CD4 origin were clearly PD $-1^{+}{ }^{42}$ Thus, at least within the LN and with the limits of these techniques, the paradigm seems to be that $\mathrm{CD}^{+} / \mathrm{PD}-1^{+} \mathrm{T}$ lymphocytes interact with $\mathrm{CD} 23^{+} / \mathrm{PD}$ $\mathrm{L1}^{+}$leukemic B cells, and this occurs specifically in the CLL LN. This crosstalk might be relevant in modulating cytokine production by $\mathrm{CD}^{+}$lymphocytes, contributing to CLL cell activation and proliferation.

PD-L1 expression by a subset of leukemic cells was maintained in the periphery, with no clear-cut association with specific markers of the disease, at least in the cohort analyzed. As in PD-1, PD-L1 might be dynamically acquired while CLL cells circulate in different environments, being exquisitely sensitive to the influence of extracellular signals and conditions. In agreement with this hypothesis, PD-L1 is inducible in lymphoid cells after treatment with IFN- $\gamma,{ }^{43}$ a cytokine with elevated levels in the serum of CLL patients. ${ }^{44}$ Within CLL LN, PD-L1 expression was higher in general and displayed two distinct patterns. The first was characterized by an intense and homogenous expression, while the second was characterized by a more focalized expression inside the PC. These characteristic patterns might indicate that PD-L1+ leukemic lymphocytes are selectively increased in residential areas, where leukemic proliferation and expansion are believed to take place. Furthermore, in vitro activation of CLL cells purified from the peripheral blood was followed by a sharp increase in the surface expression of PD-L1, expressed at high density by the majority of cells. PD-L1 is constitutively expressed at low levels by circulating normal B lymphocytes, and it is up-regulated significantly less than in CLL cells. Such different behavior could also be the result of a different ontogeny of the two populations, reflecting differentiation specific expression profiles of nuclear factors driving PD-L1 expression. ${ }^{45}$

This work also shows that a functional impairment of the PD-1/PD-L1 pathway is accompanied by an alteration in the cytokine profile of $\mathrm{T}$ lymphocytes. Preliminary in vitro data obtained by using soluble ligands and blocking antibodies to interfere with the pathway indicates that PD-1 signaling in $\mathrm{T}$ lymphocytes leads to further suppression of IFN- $\gamma$ secretion, while IL-4 appears less affected. This finding suggests that this receptor ligand pair is involved in determining the Th2 skewage typically associated with CLL and is in line with recent data indicating that PD-1 silencing restores physiological mechanisms of the immune synapse, enabling full activation of T lymphocytes. ${ }^{15}$

Further studies are needed to fully understand the role 
of PD-1/PD-L1 crosstalk in the complex interactions undergoing between CLL cells and the host $\mathrm{T}$ lymphocytes. However, considered together, the data obtained in this work provide an initial rationale for combining therapies targeting the PD-1/PD-L1 axis with other immunomodulatory agents that have shown highly promising activity in this still incurable disease.

\section{Acknowledgments}

The authors would like to thank Katiuscia Gizzi and Maria Lamusta for excellent technical assistance.

\section{Funding}

This work was supported by grants to SD from Associazione Italiana Ricerca Cancro (IG \#8590), Italian Ministries of Health (Bando Giovani Ricercatori 2008 n. GR-2008-1138053) and Education (Bando FIRB Giovani 2008 n. RBFRO8ATLH and Bando PRIN 2009 n. 2009LMEEEH_002).

\section{Authorship and Disclosures}

Information on authorship, contributions, and financial \& other disclosures was provided by the authors and is available with the online version of this article at www. haematologica.org.

\section{References}

1. Caligaris-Cappio F, Ghia P. Novel insights in chronic lymphocytic leukemia: are we getting closer to understanding the pathogenesis of the disease? J Clin Oncol. 2008;26(27):4497-503

2. Fecteau JF, Kipps TJ. Structure and function of the hematopoietic cancer niche: focus on chronic lymphocytic leukemia. Front Biosci (Schol Ed). 2012;4:61-73.

3. Pleyer L, Egle A, Hartmann TN, Greil R. Molecular and cellular mechanisms of CLL: novel therapeutic approaches. Nat Rev Clin Oncol. 2009;6(7):405-18.

4. Zenz T, Mertens D, Kuppers R, Dohner H, Stilgenbauer $\mathrm{S}$. From pathogenesis to treatment of chronic lymphocytic leukaemia. Nat Rev Cancer. 2010;10(1):37-50.

5. Walton JA, Lydyard PM, Nathwani A, Emery V, Akbar A, Glennie MJ, et al. Patients with B cell chronic lymphocytic leukaemia have an expanded population of CD4 perforin expressing T cells enriched for human cytomegalovirus specificity and an effector-memory phenotype. $\mathrm{Br} \mathrm{J}$ Haematol. 2010;148(2):274-84.

6. Nunes C, Wong R, Mason M, Fegan C, Man S, Pepper C. Expansion of a CD8(+)PD-1(+) replicative senescence phenotype in early stage CLL patients is associated with inverted CD4:CD8 ratios and disease progression. Clin Cancer Res. 2012;18(3):678-87.

7. Scrivener S, Kaminski ER, Demaine A, Prentice AG. Analysis of the expression of critical activation/interaction markers on peripheral blood $\mathrm{T}$ cells in B-cell chronic lymphocytic leukaemia: evidence of immune dysregulation. Br J Haematol. 2001;112(4):959-64.

8. Scrivener S, Goddard RV, Kaminski ER, Prentice AG. Abnormal T-cell function in Bcell chronic lymphocytic leukaemia. Leuk Lymphoma. 2003;44(3):383-9.

9. Soma LA, Craig FE, Swerdlow SH. The proliferation center microenvironment and prognostic markers in chronic lymphocytic leukemia/small lymphocytic lymphoma. Hum Pathol. 2006;37(2):152-9.

10. Patten PE, Buggins AG, Richards J, Wotherspoon A, Salisbury J, Mufti GJ, et al. CD38 expression in chronic lymphocytic leukemia is regulated by the tumor microenvironment. Blood. 2008;111(10): 5173-81.

11. Bagnara D, Kaufman MS, Calissano C, Marsilio S, Patten P, Simone R, et al. A novel adoptive transfer model of chronic lymphocytic leukemia suggests a key role for T lymphocytes in the disease. Blood.

\section{1;117:5463-72.}

12. Gonzalez-Rodriguez AP, Contesti J, Huergo-Zapico L, Lopez-Soto A, Fernandez-Guizan A, Acebes-Huerta A, et al. Prognostic significance of CD8 and CD4 $\mathrm{T}$ cells in chronic lymphocytic leukemia. Leuk Lymphoma. 2010;51(10):1829-36.

13. Gorgun G, Holderried TA, Zahrieh D, Neuberg D, Gribben JG. Chronic lymphocytic leukemia cells induce changes in gene expression of CD4 and CD8 T cells. J Clin Invest. 2005;115(7):1797-805

14. Ramsay AG, Johnson AJ, Lee AM, Gorgun G, Le Dieu R, Blum W, et al. Chronic lymphocytic leukemia $\mathrm{T}$ cells show impaired immunological synapse formation that can be reversed with an immunomodulating drug. J Clin Invest. 2008;118(7):242737 .

15. Ramsay AG, Clear AJ, Fatah R, Gribben JG Multiple inhibitory ligands induce impaired $\mathrm{T}$ cell immunological synapse function in chronic lymphocytic leukemia that can be blocked with lenalidomide. Blood. 2012; 120:1412-21.

16. Brusa D, Garetto S, Chiorino G, Scatolini M, Migliore E, Camussi G, et al. Post-apoptotic tumors are more palatable to dendritic cells and enhance their antigen cross-presentation activity. Vaccine. 2008;26 (50):6422-32.

17. Grzywnowicz M, Zaleska J, Mertens D, Tomczak W, Wlasiuk P, Kosior K, et al. Programmed death-1 and its ligand are novel immunotolerant molecules expressed on leukemic B cells in chronic lymphocytic leukemia. PLoS One. 2012;7(4):e35178.

18. Li M, Sun XH, Zhu XJ, Jin SG, Zeng ZJ, Zhou ZH, et al. HBcAg induces PD-1 upregulation on $\mathrm{CD} 4+\mathrm{T}$ cells through activation of JNK, ERK and PI3K/AKT pathways in chronic hepatitis-B-infected patients. Lab Invest. 2012;92(2):295-304

19. Shimatani K, Nakashima Y, Hattori M Hamazaki Y, Minato N. PD-1+ memory phenotype $\mathrm{CD} 4+\mathrm{T}$ cells expressing C/EBPalpha underlie $T$ cell immunodepression in senescence and leukemia. Proc Natl Acad Sci USA. 2009;106(37):15807-12.

20. Adekambi T, Ibegbu CC, Kalokhe AS, Yu T, Ray SM, Rengarajan J. Distinct Effector Memory CD4(+) T Cell Signatures in Latent Mycobacterium tuberculosis Infection, BCG Vaccination and Clinically Resolved Tuberculosis. PLoS One. 2012;7 (4):e36046.

21. Francisco LM, Sage PT, Sharpe AH. The PD-1 pathway in tolerance and autoimmunity. Immunol Rev. 2010;236:219-42.

22. Monteiro M, Evaristo C, Legrand A, Nicoletti A, Rocha B. Cartography of gene expression in CD8 single cells: novel
CCR7-subsets suggest differentiation independent of CD45RA expression. Blood. 2007;109(7):2863-70.

23. Ellis JS, Guloglu FB, Tartar DM, Hoeman CM, Haymaker CL, Cascio JA, et al. APCs expressing high levels of programmed death ligand 2 sustain the development of CD4 T cell memory. J Immunol. 2010; 185(6):3149-57.

24. Shen T, Zheng J, Liang H, Xu C, Chen X, Zhang $\mathrm{T}$, et al. Characteristics and PD-1 expression of peripheral CD4+CD127lo CD25hiFoxP3+ Treg cells in chronic HCV infected-patients. Virol J. 2011;8:279.

25. Kao C, Oestreich KJ, Paley MA, Crawford A, Angelosanto JM, Ali MA, et al. Transcription factor T-bet represses expression of the inhibitory receptor PD-1 and sustains virus-specific $\mathrm{CD} 8+\mathrm{T}$ cell responses during chronic infection. Nat Immunol. 2011;12(7):663-71.

26. Dicker F, Schnittger S, Haferlach T, Kern W, Schoch C. Immunostimulatory oligonucleotide-induced metaphase cytogenetics detect chromosomal aberrations in $80 \%$ of CLL patients: A study of 132 CLL cases with correlation to FISH, IgVH status, and CD38 expression. Blood. 2006;108(9):3152-60.

27. Dorfman DM, Brown JA, Shahsafaei A, Freeman GJ. Programmed death-1 (PD-1) is a marker of germinal center-associated $\mathrm{T}$ cells and angioimmunoblastic T-cell lymphoma. Am J Surg Pathol. 2006;30(7):80210.

28. Xerri L, Chetaille B, Serriari N, Attias C, Guillaume Y, Arnoulet C, et al. Programmed death 1 is a marker of angioimmunoblastic T-cell lymphoma and B-cell small lymphocytic lymphoma/chronic lymphocytic leukemia. Hum Pathol. 2008;39(7):1050-8.

29. Porakishvili N, Roschupkina T, Kalber $T$ Jewell AP, Patterson K, Yong K, et al. Expansion of CD4+ T cells with a cytotoxic phenotype in patients with B-chronic lymphocytic leukaemia (B-CLL). Clin Exp Immunol. 2001;126(1):29-36.

30. Podhorecka M, Dmoszynska A, Rolinski J Wasik E. T type 1/type 2 subsets balance in B-cell chronic lymphocytic leukemia--the three-color flow cytometry analysis. Leuk Res. 2002;26(7):657-60.

31. Schreiber RD, Old LJ, Smyth MJ. Cancer immunoediting: integrating immunity's roles in cancer suppression and promotion. Science. 2011:331(6024):1565-70.

32. Flies DB, Sandler BJ, Sznol M, Chen L. Blockade of the B7-H1/PD-1 pathway for cancer immunotherapy. Yale J Biol Med. 2011;84(4):409-21.

33. Wang S, Chen L. Immunobiology of cancer therapies targeting CD137 and B7-H1/PD-1 
cosignal pathways. Curr Top Microbiol Immunol. 2011;344:245-67.

34. Brahmer JR, Tykodi SS, Chow LQ, Hwu WJ, Topalian SL, Hwu P, et al. Safety and activity of anti-PD-L1 antibody in patients with advanced cancer. $N$ Engl J Med. 2012;366(26):2455-65.

35. Topalian SL, Hodi FS, Brahmer JR, Gettinger SN, Smith DC, McDermott DF, et al. Safety, activity, and immune correlates of anti-PD-1 antibody in cancer. $\mathrm{N}$ Engl J Med. 2012;366(26):2443-54.

36. Good-Jacobson KL, Szumilas CG, Chen L, Sharpe AH, Tomayko MM, Shlomchik MJ. $\mathrm{PD}-1$ regulates germinal center $\mathrm{B}$ cell survival and the formation and affinity of long-lived plasma cells. Nature immunology. 2010;11(6):535-42.

37. Shimauchi T, Kabashima K, Nakashima D, Sugita K, Yamada Y, Hino R, et al. Augmented expression of programmed death-1 in both neoplastic and non-neoplastic CD4+ T-cells in adult T-cell leukemia/lymphoma. Int J Cancer. 2007; 121(12):2585-90.

38. Yamamoto $R$, Nishikori M, Kitawaki $T$ Sakai T, Hishizawa M, Tashima M, et al. PD-1-PD-1 ligand interaction contributes to immunosuppressive microenvironment of Hodgkin lymphoma. Blood. 2008;111(6): 3220-4

39. Richendollar BG, Pohlman B, Elson P, Hsi ED. Follicular programmed death 1-positive lymphocytes in the tumor microenvironment are an independent prognostic factor in follicular lymphoma. Hum Pathol. 2011;42(4):552-7.

40. Mazanet MM, Hughes CC. B7-H1 is expressed by human endothelial cells and suppresses $\mathrm{T}$ cell cytokine synthesis. J Immunol. 2002;169(7):3581-8.

41. Rodig N, Ryan T, Allen JA, Pang H, Grabie $\mathrm{N}$, Chernova $\mathrm{T}$, et al. Endothelial expression of PD-L1 and PD-L2 down-regulates CD8+ T cell activation and cytolysis. Eur J Immunol. 2003;33(11):3117-26.
42. Dorfman DM, Shahsafaei A. CD200 (OX-2 membrane glycoprotein) is expressed by follicular $\mathrm{T}$ helper cells and in angioimmunoblastic T-cell lymphoma. Am J Surg Pathol. 2011;35(1):76-83.

43. Chen J, Feng Y, Lu L, Wang H, Dai L, Li Y et al. Interferon-gamma-induced PD-L1 surface expression on human oral squamous carcinoma via PKD2 signal pathway. Immunobiology. 2012;217(4):385-93.

44. Samy N, Abd El-Maksoud MD, Mousa TE, El-Mezayen HA, Shaalan M. Potential role of serum level of soluble CD44 and IFNgamma in B-cell chronic lymphocytic leukemia. Med Oncol. 2011;28 (1 Suppl):S471-5.

45. Green MR, Rodig S, Juszczynski P, Ouyang J, Sinha P, O'Donnell E, et al. Constitutive AP-1 activity and EBV infection induce PDL1 in Hodgkin lymphomas and posttransplant lymphoproliferative disorders: implications for targeted therapy. Clin Cancer Res. 2012;18(6):1611-8. 